\title{
Supersymmetric Chern-Simons Theory and Supersymmetric Quantum Hall Liquid
}

\author{
Kazuki Hasebe \\ Department of General Education, \\ Takuma National College of Technology, \\ Takuma-cho, Mitoyo-city, Kagawa 769-1192, Japan \\ Email: hasebe@dg.takuma-ct.ac.jp
}

\begin{abstract}
We develop a supersymmetric extension of Chern-Simons theory and Chern-Simons-LandauGinzburg theory for the supersymmetric quantum Hall liquid. Supersymmetric counterparts of topological and gauge structures peculiar to the Chern-Simons theory are inspected in the supersymmetric Chern-Simons theory. We also explore an effective field theoretical description for the supersymmetric quantum Hall liquid. The key observation is the the charge-flux duality. Based on the duality, we derive a dual supersymmetric Chern-Simons-Landau-Ginzburg theory, and discuss physical properties of the topological excitations in the supersymmetric quantum Hall liquid.
\end{abstract}

\section{INTRODUCTION}

In recent years, concepts of quantum Hall effect (QHE), which was believed to be formulated only in two-dimensional space, have been dramatically changing, initiated by the success of the construction of the fourdimensional QHE [1]. Since then, further generalizations of the QHE have been explored, in even higher dimensions [2-6] and in $q$-deformed systems [7]. More recently, inspired by the developments of the non-anticommutative geometry [8-10], supersymmetric (SUSY) extensions of QH liquids were proposed on noncommutative supermanifolds, such as a fuzzy supersphere [11] and a noncommutative superplane [12]. The analyses of the SUSY QHE revealed some of the novel physical consequences of the non-anticommutative geometry. The SUSY QH liquids exhibit natural SUSY counterparts of the mathematical and physical features peculiar to the original QH liquids, for instance, noncommutative geometry, fractionally charged excitations, Hall orthogonality and $W_{\infty}$ symmetry. The SUSY Landau problems on other analogous noncommutative supermanifolds, such as fuzzy $\mathbb{C} P^{(n \mid m)}$, were studied in Ref.[13], a higher SUSY Landau problem was also reported in Ref.[14], and a SUSY extension of the $\mathrm{QH}$ matrix model was constructed in Ref.[15].

One of the most amazing incidents of QHE is the emergence of the Chern-Simons (CS) topological field theory in a low energy sector. The CS flux attachment to electrons induces the statistical transmutation from fermion to (composite) boson, and the QH states are regarded as "superfluid" states of composite bosons. The ChernSimons-Landau-Ginzburg (CSLG) theory describes low energy phenomenology in the QHE [16-18]. Apart from relations to QHE, the CS theory is important of its own right in a field theoretical point of view [19]. It is quite interesting that such a novel field theory appears from mysterious many-body effects, and well describes collective phenomena of a real condensed matter system.

In this paper, we develop a SUSY extension of the CS theory and CSLG theory with $O S p(1 \mid 2)$ global symmetry. The SUSY CS theory demonstrates natural SUSY extensions of the topological and gauge features peculiar to the original CS theory. We also explore the CSLG description for the SUSY QH liquid. We show the existence of the charge-flux duality in the SUSY QH system, and derive the dual representation for the SUSY CSLG theory. Based on the dual description, physical properties of the topological excitations in SUSY QH liquids are discussed.

This paper is organized as follows. In Section II, we briefly review the mathematical background for the SUSY QH liquid. In Section III, we present a Lagrange formalism of the one-particle mechanics on a supersphere in the presence of the supermonopole background. In Section IV, the SUSY CS theory is constructed and its field theoretical properties are inspected. In Section V, the charge-flux duality in the SUSY system is explored. With the use of the dual SUSY CSLG description, we discuss physical properties of the topological excitations. Section VI is devoted to summary and discussions. In Appendix A, several useful formulas on supermatrix are summarized. In Appendix B, we present the super Jacobi and Bianchi identities.

\section{THE SUPER HOPF MAP AND THE SUPERMONOPOLE}

In this section, we review the super Hopf map and the supermonopole $[20,21]$ used in the set-up of the supersymmetric QH liquid. First, we introduce the $O S p(1 \mid 2)$ supergroup, whose generators are given by $l_{a}(a=x, y, z)$, which are Grassmann even, and $l_{\alpha}$ $\left(\alpha=\theta_{1}, \theta_{2}\right)$, which are Grassmann odd. They satisfy the following graded algebras:

$$
\begin{aligned}
& {\left[l_{a}, l_{b}\right]=i \epsilon_{a b c} l_{c},} \\
& {\left[l_{a}, l_{\alpha}\right]=\frac{1}{2}\left(\sigma_{a}\right)_{\beta \alpha} l_{\beta},} \\
& \left\{l_{\alpha}, l_{\beta}\right\}=\frac{1}{2}\left(C \sigma_{a}\right)_{\alpha \beta} l_{a} .
\end{aligned}
$$


The fundamental representations of the $O S p(1 \mid 2)$ generators are given by

$$
l_{a}=\frac{1}{2}\left(\begin{array}{cc}
\sigma_{a} & 0 \\
0 & 0
\end{array}\right), \quad l_{\alpha}=i \frac{1}{2}\left(\begin{array}{cc}
0 & \tau_{\alpha} \\
\left(C \tau_{\alpha}\right)^{t} & 0
\end{array}\right),
$$

where $\left\{\sigma_{a}\right\}$ are Pauli matrices, $C$ is the charge conjugation matrix given by $C=i \sigma_{2}$ and $\tau_{1}=(1,0)^{t}$, $\tau_{2}=(0,1)^{t}$. The general element of the $\operatorname{OSp}(1 \mid 2) \mathrm{su}-$ pergroup (strictly speaking $U O S p(1 \mid 2)$ supergroup), is parameterized as

$$
g=\left(\begin{array}{ccc}
u & -v^{*} & \eta^{*} u-\eta v^{*} \\
v & u^{*} & \eta u^{*}+\eta^{*} v \\
\eta & -\eta^{*} & 1-\eta^{*} \eta
\end{array}\right)
$$

where $u$ and $v$ are Grassmann even complex parameters, $\eta$ is a Grassmann odd parameter, and they are chosen to satisfy the constraint $u^{*} u+v^{*} v-\eta^{*} \eta=1$. With this constraint, one may see that $g$ satisfies the following conditions

$$
\operatorname{sdet}(g)=u^{*} u+v^{*} v-\eta^{*} \eta=1
$$

and

$$
g^{\ddagger} g=g g^{\ddagger}=1 \text {. }
$$

The definitions of the super-determinant sdet, and the super-adjoint $\ddagger$ are given by Eqs.(A2) and (A3), respectively. (Our definition of the super-adjoint is different from the conventional one.)

The super Hopf map is given by the mapping from $O S p(1 \mid 2)$ element $g$ to the coordinates $\left(x_{a}, \theta_{\alpha}\right)$ on the supersphere $S^{2 \mid 2}$

$$
g \rightarrow g l_{3} g^{\ddagger}=x_{a} l_{a}+i C_{\alpha \beta} \theta_{\alpha} l_{\beta} .
$$

Taking square and supertrace on both sides (See Eqs.(A4) and (A5)), it is easily checked that $\left(x_{a}, \theta_{\alpha}\right)$ satisfy the constraint

$$
x_{a}^{2}+C_{\alpha \beta} \theta_{\alpha} \theta_{\beta}=1,
$$

which defines the supersphere with unit radius. With the use of the Hopf spinor $\psi=(u, v, \eta)^{t},\left(x_{a}, \theta_{\alpha}\right)$ in Eq.(2.6) are concisely represented as

$$
\begin{aligned}
& x_{a}=2 \psi^{\dagger} l_{a} \psi, \\
& \theta_{\alpha}=-2 i \psi^{\dagger} l_{\alpha} \psi,
\end{aligned}
$$

where $\psi^{\dagger} \equiv\left(u^{*}, v^{*}, \eta^{*}\right)$. Since $\left(x_{a}, \theta_{\alpha}\right)$ are invariant under the $U(1)$ transformation

$$
g \rightarrow g \cdot e^{2 i \alpha l_{3}}
$$

or

$$
\psi \rightarrow e^{i \alpha} \psi
$$

the supersphere is given by the coset $S^{2 \mid 2}=$ $O S p(1 \mid 2) / U(1)$, and $U(1)$ fibre is defined on $S^{2 \mid 2}$. The connection of the $U(1)$ fiber is given by

$$
A=-i \operatorname{str}\left(l_{3} g^{\ddagger} d g\right)=-i \frac{1}{2}\left(\psi^{\ddagger} d \psi-d \psi^{\ddagger} \psi\right),
$$

where $\psi^{\ddagger} \equiv\left(u^{*}, v^{*},-\eta^{*}\right)$. Under the $U(1)$ transformation (2.9) or (2.10), as expected, $A$ is transformed as

$$
A \rightarrow A+d \alpha \text {. }
$$

Inverting the super Hopf map (2.8) from $\left(x_{a}, \theta_{\alpha}\right)$ to $\psi$, the super Hopf spinor is expressed as [21],

$$
\psi=\left(\begin{array}{c}
\sqrt{\frac{1+x_{3}}{2}}\left(1-\frac{1}{4\left(1+x_{3}\right)} \theta C \theta\right) \\
\frac{x_{1}+i x_{2}}{\sqrt{2\left(1+x_{3}\right)}}\left(1+\frac{1}{4\left(1+x_{3}\right)} \theta C \theta\right) \\
\frac{1}{\sqrt{2\left(1+x_{3}\right)}}\left(\left(1+x_{3}\right) \theta_{1}+\left(x_{1}+i x_{2}\right) \theta_{2}\right)
\end{array}\right) .
$$

Using this explicit form, the supermonopole gauge fields $A=d x_{a} A_{a}+d \theta_{\alpha} A_{\alpha}$ are calculated as

$$
\begin{aligned}
& A_{a}=-\frac{I}{2} \epsilon_{a b 3} \frac{x_{b}}{1+x_{3}}\left(1+\frac{2+x_{3}}{2\left(1+x_{3}\right)} \theta C \theta\right), \\
& A_{\alpha}=-i \frac{I}{2}\left(\sigma_{a} C\right)_{\alpha \beta} x_{a} \theta_{\beta},
\end{aligned}
$$

with $I=1$. The supermonopole gauge fields with quantized charges take the same form as Eqs.(2.14) with the integer $I$. With the use of super gauge fields (2.14), the supermonopole field strengths are obtained as

$$
\begin{aligned}
& F_{a b}=\frac{I}{2} \epsilon_{a b c} x_{c}\left(1+\frac{3}{2} \theta C \theta\right), \\
& F_{a \alpha}=-i \frac{I}{2}\left(\delta_{a b}-3 x_{a} x_{b}\right)\left(\theta \sigma_{b} C\right)_{\alpha}, \\
& F_{\alpha \beta}=-i I\left(\sigma_{a} C\right)_{\alpha \beta} x_{a}\left(1+\frac{3}{2} \theta C \theta\right),
\end{aligned}
$$

where we used the definition of the super field strengths

$$
\begin{aligned}
& F_{a b}=\partial_{a} A_{b}-\partial_{b} A_{a}, \\
& F_{a \alpha}=\partial_{a} A_{\alpha}-\partial_{\alpha} A_{a}, \\
& F_{\alpha \beta}=\partial_{\alpha} A_{\beta}+\partial_{\beta} A_{\alpha} .
\end{aligned}
$$

\section{ONE-PARTICLE ON THE SUPERSPHERE IN THE SUPERMONOPOLE BACKGROUND}

Before discussing the many-body system, we consider one-particle mechanics on the supersphere with unit radius in the supermonopole background. The supermonopole is set at the center of the supersphere. The one-particle Lagrangian is given by

$$
L=\frac{m}{2}\left(\dot{x}_{a}^{2}+C_{\alpha \beta} \dot{\theta_{\alpha}} \dot{\theta_{\beta}}\right)+\dot{x}_{a} A_{a}+\dot{\theta}_{\alpha} A_{\alpha}-V,
$$


where $V$ is the external electric potential, $\left(A_{a}, A_{\alpha}\right)$ are supermonopole gauge fields (2.14), and $\left(x_{a}, \theta_{\alpha}\right)$ satisfy the constraint (2.7). Introducing a Lagrange multiplier $\lambda$, the equations of motion are derived as

$$
\begin{aligned}
& m \ddot{x}_{a}=F_{a b} \dot{x}_{b}-F_{a \alpha} \dot{\theta}_{\alpha}+E_{a}+\lambda x_{a}, \\
& m \ddot{\theta}_{\alpha}=C_{\alpha \beta}\left(F_{a \beta} \dot{x}_{a}+F_{\beta \gamma} \dot{\theta}_{\gamma}\right)+E_{\alpha}+\lambda \theta_{\alpha},
\end{aligned}
$$

where $E_{a}=-\partial_{a} V, E_{\alpha}=C_{\alpha \beta} \partial_{\beta} V$, and $\left(F_{a b}, F_{a \alpha}, F_{\alpha \beta}\right)$ are supermonopole gauge fields (2.15). From Eqs.(2.7) and (3.2), $\lambda$ is eliminated as

$$
\lambda=-m\left(\dot{x}_{a}^{2}+C_{\alpha \beta} \dot{\theta}_{\alpha} \dot{\theta}_{\beta}\right)-\left(E_{a} x_{a}+C_{\alpha \beta} E_{\alpha} \theta_{\beta}\right) .
$$

Inserting this explicit form of $\lambda$ to Eqs.(3.2), we obtain selfcontained equations of motion. Though it is quite nontrivial to solve such nonlinear equations, we may discuss local motions of the particle of our interest.

The center-of-mass coordinates $\left(X_{a}, \Theta_{\alpha}\right)$ are defined as

$$
\begin{aligned}
& X_{a}=x_{a}-\frac{2}{I} \Lambda_{a}, \\
& \Theta_{\alpha}=\theta_{\alpha}-\frac{2}{I} \Lambda_{\alpha},
\end{aligned}
$$

where $\left(\Lambda_{a}, \Lambda_{\alpha}\right)$ represent the $O S p(1 \mid 2)$ angular momenta of the particle

$$
\begin{aligned}
& \Lambda_{a}=m \epsilon_{a b c} x_{b} \dot{x}_{c}+i \frac{m}{2} \theta_{\alpha}\left(\sigma_{a} C\right)_{\alpha \beta} \dot{\theta}_{\beta}, \\
& \Lambda_{\alpha}=i \frac{m}{2} x_{a}\left(\sigma_{a}\right)_{\beta \alpha} \dot{\theta}_{\beta}-i \frac{m}{2} \theta_{\beta}\left(\sigma_{a}\right)_{\beta \alpha} \dot{x}_{a} .
\end{aligned}
$$

In the lowest Landau level (LLL) limit (which is realized at $m \rightarrow 0)$, the particle coordinates $\left(x_{a}, \theta_{\alpha}\right)$ are reduced to the center-of-mass coordinates $\left(X_{a}, \Theta_{\alpha}\right)$. With the use of Eqs.(3.2), the constraint (2.7), and its derivative $\dot{x}_{a} x_{a}+C_{\alpha \beta} \dot{\theta}_{\alpha} \theta_{\beta}=0$, the velocities of the center-of-mass coordinates are derived as

$$
\begin{aligned}
\dot{X}_{a} & =-\frac{I}{2} \epsilon_{a b c} x_{b} E_{c}-i \frac{I}{4} \theta_{\alpha}\left(\sigma_{a} C\right)_{\alpha \beta} E_{\beta}, \\
\dot{\Theta}_{\alpha} & =-i \frac{I}{4}\left(\sigma_{a}\right)_{\beta \alpha} x_{a} E_{\beta}+i \frac{I}{4}\left(\sigma_{a}\right)_{\beta \alpha} \theta_{\beta} E_{a} .
\end{aligned}
$$

In the presence of the magnetic field, a charged particle performs a drift motion, where the center-of-mass coordinates move perpendicularly to the direction of the applied electric fields. From Eqs.(3.6), we confirm such orthogonality in the SUSY sense:

$$
E_{a} \dot{X}_{a}+C_{\alpha \beta} E_{\alpha} \dot{\Theta}_{\beta}=0 .
$$

Meanwhile, from Eqs.(3.2), the particle velocities and the electric fields are related as

$$
E_{a} \dot{x}_{a}+C_{\alpha \beta} E_{\alpha} \dot{\theta}_{\beta}=m\left(\dot{x}_{a} \ddot{x}_{a}+C_{\alpha \beta} \dot{\theta}_{\alpha} \ddot{\theta}_{\beta}\right) .
$$

In the LLL limit, from Eq.(3.8), one may find the super Hall orthogonality

$$
E_{a} \dot{x}_{a}+C_{\alpha \beta} E_{\alpha} \dot{\theta}_{\beta}=0,
$$

which is consistent with the one obtained in the SUSY noncommutative formalism [11].

Without electric fields, from Eqs.(3.6), the velocities of the center-of-mass coordinates vanish

$$
\dot{X}_{a}=\dot{\Theta}_{\alpha}=0,
$$

and, from Eq.(3.8), the accelerations and velocities become orthogonal

$$
\dot{x}_{a} \ddot{x}_{a}+C_{\alpha \beta} \dot{\theta}_{\alpha} \ddot{\theta}_{\beta}=0 .
$$

Under the perpendicular magnetic field, a charged particle performs a cyclotron motion around its center-of-mass coordinates due to the Lorentz force. Equations (3.10) and (3.11) demonstrate this observation in the SUSY sense.

In the planar limit $\left(x_{3} \approx 1\right)$, the one-particle Lagrangian (3.1) is reduced to

$$
L=\frac{m}{2}\left(\dot{x}_{i}^{2}+C_{\alpha \beta} \dot{\theta}_{\alpha} \dot{\theta}_{\beta}\right)-\frac{B}{2} \epsilon_{i j} \dot{x}_{i} x_{j}-i B\left(\sigma_{1}\right)_{\alpha \beta} \dot{\theta}_{\alpha} \theta_{\beta},
$$

where $B=I / 2$. The canonical momenta are obtained as

$$
\begin{aligned}
& p_{i}=\frac{\partial}{\partial \dot{x}_{i}} L=m \dot{x}_{i}-\frac{B}{2} \epsilon_{i j} x_{j}, \\
& p_{\alpha}=\frac{\partial}{\partial \dot{\theta}_{\alpha}} L=m C_{\alpha \beta} \dot{\theta}_{\beta}-i B\left(\sigma_{1}\right)_{\alpha \beta} \theta_{\beta} .
\end{aligned}
$$

In the LLL limit, from the commutation relations $\left[x_{i}, p_{j}\right]=i \delta_{i j}$ and $\left\{\theta_{\alpha}, p_{\beta}\right\}=i \delta_{\alpha \beta}$, we obtain the algebras on the noncommutative superplane

$$
\begin{aligned}
& {\left[x_{i}, x_{j}\right]=-i \frac{1}{B} \epsilon_{i j},} \\
& \left\{\theta_{\alpha}, \theta_{\beta}\right\}=-\frac{1}{2 B}\left(\sigma_{1}\right)_{\alpha \beta} .
\end{aligned}
$$

These SUSY noncommutative algebras bring novel physics to planar SUSY quantum Hall systems [12].

\section{SUSY CS THEORY}

It is well known that CS theories are defined in spaces with odd dimensions. With the CS coupling constant $\kappa$, the CS Lagrangian in 3-dimensional space is given by

$$
\mathcal{L}_{C S}=\frac{\kappa}{4} \epsilon_{a b c} A_{a} F_{b c} .
$$

In the following, we modify $\mathcal{L}_{C S}$ to be invariant under the $O S p(1 \mid 2)$ global supersymmetry.

As a preliminary, we summarize the $O S p(1 \mid 2)$ transformations of the super gauge fields. The derivative expressions for the $O S p(1 \mid 2)$ generators are

$$
\begin{aligned}
& L_{a}=-i \epsilon_{a b c} x_{b} \partial_{c}+\frac{1}{2} \theta_{\alpha}\left(\sigma_{a}\right)_{\alpha \beta} \partial_{\beta}, \\
& L_{\alpha}=\frac{1}{2} x_{a}\left(C \sigma_{a}\right)_{\alpha \beta} \partial_{\beta}-\frac{1}{2} \theta_{\beta}\left(\sigma_{a}\right)_{\beta \alpha} \partial_{a} .
\end{aligned}
$$


With the use of the Grassmann odd generators $L_{\alpha}$, the super charge is constructed as

$$
Q=L_{\alpha} \xi_{\alpha}
$$

where $\xi_{\alpha}$ are Grassmann odd parameters. The super transformations of $\left(x_{a}, \theta_{\alpha}\right)$ read

$$
\begin{aligned}
\delta_{\xi} x_{a} & =\left[Q, x_{a}\right]=\frac{1}{2} \theta \sigma_{a} \xi, \\
\delta_{\xi} \theta_{\alpha} & =\frac{1}{2} x_{a}\left(C \sigma_{a} \xi\right)_{\alpha} .
\end{aligned}
$$

Similarly, the super gauge fields and the super field strengths are transformed as

$$
\begin{aligned}
\delta_{\xi} A_{a} & =-\frac{1}{2} A_{\alpha}\left(C \sigma_{a}\right)_{\alpha \beta} \xi_{\beta}, \\
\delta_{\xi} A_{\alpha} & =\frac{1}{2} A_{a}\left(\sigma_{a} \xi\right)_{\alpha},
\end{aligned}
$$

and

$$
\begin{aligned}
\delta_{\xi} F_{a b} & =-\frac{1}{2} F_{a \alpha}\left(C \sigma_{b} \xi\right)_{\alpha}+\frac{1}{2} F_{b \alpha}\left(C \sigma_{a} \xi\right)_{\alpha}, \\
\delta_{\xi} F_{a \alpha} & =\frac{1}{2} F_{a b}\left(\sigma_{b} \xi\right)_{\alpha}+\frac{1}{2} F_{\alpha \beta}\left(C \sigma_{a} \xi\right)_{\beta}, \\
\delta_{\xi} F_{\alpha \beta} & =-\frac{1}{2} F_{a \alpha}\left(\sigma_{a} \xi\right)_{\beta}-\frac{1}{2} F_{a \beta}\left(\sigma_{a} \xi\right)_{\alpha} .
\end{aligned}
$$

It is noted that 12-dimensional representations $\left(F_{a b}, F_{a \alpha}, F_{\alpha \beta}\right)$ are not irreducible representations but irreducibly decomposed into $\mathbf{7} \oplus \mathbf{5}$ representations. The $\mathbf{5}$ dimensional irreducible representations $\left(F_{a}, F_{\alpha}\right)$, which we call the super vector field strengths, are constructed as

$$
\begin{aligned}
& F_{a}=\frac{1}{2} \epsilon_{a b c} F_{b c}+i \frac{1}{4}\left(C \sigma_{a}\right)_{\alpha \beta} F_{\alpha \beta}, \\
& F_{\alpha}=-i \frac{1}{2}\left(C \sigma_{a}\right)_{\alpha \beta} F_{a \beta} .
\end{aligned}
$$

It is easy to check that, under the super transformations (4.6), $\left(F_{a}, F_{\alpha}\right)$ form a super multiplet

$$
\begin{aligned}
\delta_{\xi} F_{a} & =\frac{1}{2} F_{\alpha}\left(\sigma_{a} \xi\right)_{\alpha}, \\
\delta_{\xi} F_{\alpha} & =\frac{1}{2} F_{a}\left(C \sigma_{a} \xi\right)_{\alpha},
\end{aligned}
$$

and satisfy the scalar super Bianchi identity

$$
\partial_{a} F_{a}+\partial_{\alpha} F_{\alpha}=0
$$

where we used the identities $\epsilon_{a b c} \partial_{a} F_{b c}=0$ and $\left(C \sigma_{a}\right)_{\alpha \beta}\left(2 \partial_{\alpha} F_{\beta a}-\partial_{a} F_{\alpha \beta}\right)=0$, which are obtained from the super Bianchi identities (B2) and (B4).

From the inner product of $\left(A_{a}, A_{\alpha}\right)$ and $\left(F_{a}, F_{\alpha}\right)$, an $O S p(1 \mid 2)$ singlet is constructed as

$$
\mathcal{L}_{s C S}=\frac{\kappa}{2}\left(A_{a} F_{a}+A_{\alpha} F_{\alpha}\right)
$$

which we adopt as the SUSY CS Lagrangian. In terms of $\left(F_{a b}, F_{a \alpha}, F_{\alpha \beta}\right), \mathcal{L}_{s C S}$ is rewritten as

$$
\begin{aligned}
\mathcal{L}_{s C S} \\
=\frac{\kappa}{4}\left(\epsilon_{a b c} A_{a} F_{b c}-i\left(C \sigma_{a}\right)_{\alpha \beta} A_{\alpha} F_{a \beta}+\frac{i}{2}\left(C \sigma_{a}\right)_{\alpha \beta} A_{a} F_{\alpha \beta}\right) \\
=\frac{\kappa}{2}\left(\epsilon_{a b c} A_{a} \partial_{b} A_{c}-\frac{i}{2}\left(C \sigma_{a}\right)_{\alpha \beta} A_{\alpha} \partial_{a} A_{\beta}+i\left(C \sigma_{a}\right)_{\alpha \beta} A_{\alpha} \partial_{\beta} A_{a}\right) \\
\quad+(\text { total fermionic derivative term }) .
\end{aligned}
$$

Though either term in $\mathcal{L}_{s C S}$ is not $O S p(1 \mid 2)$ singlet, $\mathcal{L}_{s C S}$ is invariant in total. To respect the $O S p(1 \mid 2)$ global symmetry, the basespace for the SUSY CS Lagrangian is given by $\mathbb{R}^{3 \mid 2}$, whose volume element is $d^{3} x d^{2} \theta$. Thus, we have obtained the SUSY CS action invariant under the $O S p(1 \mid 2)$ super transformation, while it is not invariant under general super coordinate transformations. Namely, the form of our SUSY CS action critically depends on the particular choice of the coordinates and the background manifold. Then, in this sense, our SUSY CS theory is not a topological field theory on supermanifolds. (Topological field theories do not depend on the background metric and are invariant under general coordinate transformations.) However, our SUSY CS theory still inherits topological natures peculiar to the original CS theory and manifests them in a SUSY sense as we shall see in Subsections IV B and IV D. Some comments are added further. One may find "various kinds" of SUSY CS theories in literature. For instance, in Ref.[22], the SUSY CS theory is referred to as the CS theory with $O S p(1 \mid 2)$ gauge symmetry. In Ref.[23], the SUSY CS theory is referred to as the CS theory coupled to super matter currents. In the above, we derived a new SUSY CS Lagrangian, which possesses the $O S p(1 \mid 2)$ global supersymmetry. The matrix analogue of our SUSY CS Lagrangian is found in Ref.[24], where the SUSY CS term plays a crucial role for the realization of fuzzy superspheres in the supermatrix model. In the following subsections, we investigate field theoretical aspects of our SUSY CS theory.

\section{A. $U(1)$ gauge symmetry}

The original CS Lagrangian (4.1) is invariant under the $U(1)$ gauge transformation up to a total derivative term. Similarly, under the $U(1)$ gauge transformation with the gauge function $\Lambda$

$$
\left(A_{a}, A_{\alpha}\right) \rightarrow\left(A_{a}, A_{\alpha}\right)+\left(\partial_{a} \Lambda, \partial_{\alpha} \Lambda\right)
$$

$\mathcal{L}_{s C S}$ only yields the total derivative terms

$$
\begin{aligned}
\delta \mathcal{L}_{s C S}= & \frac{\kappa}{2}\left(\partial_{a}\left(\Lambda F_{a}\right)+\partial_{\alpha}\left(\Lambda F_{\alpha}\right)\right) \\
= & \frac{\kappa}{4}\left(\partial_{a}\left(\Lambda \epsilon_{a b c} F_{b c}\right)-i \partial_{\alpha}\left(\Lambda\left(C \sigma_{a}\right)_{\alpha \beta} F_{a \beta}\right)\right. \\
& \left.+\frac{i}{2} \partial_{a}\left(\Lambda\left(C \sigma_{a}\right)_{\alpha \beta} F_{\alpha \beta}\right)\right)
\end{aligned}
$$


where the scalar Bianchi identity (4.9) was used. Thus, $\mathcal{L}_{s C S}$ is invariant under the $U(1)$ gauge transformation when the boundary contributions are neglected.

\section{B. Coupling with matter}

Next, we investigate the SUSY CS theory with matter fields, by adding the interaction term

$$
\mathcal{L}_{I}=A_{a} J_{a}+A_{\alpha} J_{\alpha}
$$

to $\mathcal{L}_{s C S}$, where $\left(J_{a}, J_{\alpha}\right)$ are the matter currents that form a super multiplet

$$
\begin{aligned}
\delta_{\xi} J_{a} & =\frac{1}{2} J_{\alpha}\left(\sigma_{a} \xi\right)_{\alpha}, \\
\delta_{\xi} J_{\alpha} & =\frac{1}{2} J_{a}\left(C \sigma_{a} \xi\right)_{\alpha} .
\end{aligned}
$$

The equations of motion for SUSY CS fields are given by

$$
\begin{aligned}
& \kappa F_{a}=-J_{a}, \\
& \kappa F_{\alpha}=-J_{\alpha} .
\end{aligned}
$$

It is obvious that the current conservation

$$
\partial_{a} J_{a}+\partial_{\alpha} J_{\alpha}=0
$$

is compatible with the scalar super Bianchi identity (4.9). Regarding the 3rd bosonic axis as the temporal direction, we consider point particles coupled to the SUSY CS fields in Wick-rotated Euclidean super space-time $\mathbb{R}^{3 \mid 2}$. The Lagrangian is given by

$L=\sum_{p} \frac{m}{2}\left(\dot{x}_{i}^{p} \dot{x}_{i}^{p}+C_{\alpha \beta} \dot{\theta}_{\alpha}^{p} \dot{\theta}_{\beta}^{p}\right)+\int d^{2} x d^{2} \theta \mathcal{L}_{I}+\int d^{2} x d^{2} \theta \mathcal{L}_{s C S}$,

where $\left(x_{i}^{p}, \theta_{\alpha}^{p}\right)(i=1,2)$ denotes the position of the $p$-th particle, and the matter currents are

$$
\begin{aligned}
& J_{a}(x, \theta)=\sum_{p} \dot{x}_{a}^{p} \delta^{2}\left(x-x^{p}\right) \delta^{2}\left(\theta-\theta^{p}\right), \\
& J_{\alpha}(x, \theta)=\sum_{p} \dot{\theta}_{a}^{p} \delta^{2}\left(x-x^{p}\right) \delta^{2}\left(\theta-\theta^{p}\right) .
\end{aligned}
$$

The equations of motion for the $p$-th particle are derived as

$$
\begin{aligned}
& m \ddot{x}_{i}^{p}=F_{i a} \dot{x}_{a}^{p}-F_{i \alpha} \dot{\theta}_{\alpha}^{p}, \\
& m \ddot{\theta}_{\alpha}^{p}=C_{\alpha \beta}\left(F_{a \beta} \dot{x}_{a}^{p}+F_{\beta \gamma} \dot{\theta}_{\gamma}^{p}\right) .
\end{aligned}
$$

With the use of Eqs.(4.16), the equations of motion (4.20) are reduced to those of the free particle

$$
\begin{aligned}
\ddot{x}_{i}^{p} & =0, \\
\ddot{\theta}_{\alpha}^{p} & =0 .
\end{aligned}
$$

Thus, the SUSY CS gauge fields never affect the dynamical motion of the matter particles in bulk. This is a consequence of the topological nature of the SUSY CS theory.

\section{SUSY Hopf term}

The phase interaction between two anyons is described by the non-local Hopf term

$$
\begin{aligned}
\mathcal{L}_{\text {Hopf }} & =-\frac{1}{2 \kappa} \int d^{3} x \int d^{3} y \epsilon_{a b c} J_{a}(x) \frac{x_{b}-y_{b}}{|x-y|^{3}} J_{c}(y) \\
& =-\frac{1}{2 \kappa} J \frac{\epsilon \partial}{\partial^{2}} J,
\end{aligned}
$$

where $(\epsilon \partial)_{a b}=\epsilon_{a b c} \partial_{c}, \partial^{2}=\partial_{a}^{2}$ and $J_{a}$ represents the anyon current. Introducing the CS gauge fields, the Hopf interaction is rewritten as the CS local interaction $\frac{\kappa}{2} A \epsilon \partial A+A J$. (Integrating out the CS fields, we obtain the Hopf term (4.22).) The Hopf term corresponds to the topological invariant of the two closed (anyon) loops, known as Gauss linking number. It would be quite interesting to see the SUSY extension of the Hopf term by integrating out the SUSY CS gauge fields in the SUSY CS Lagrangian. For later convenience, we rewrite the SUSY CS Lagrangian in matrix representation

$$
\begin{aligned}
\mathcal{L} & =\mathcal{L}_{s C S}+\mathcal{L}_{I} \\
& =\frac{\kappa}{2}\left(\begin{array}{l}
A \\
\mathcal{A}
\end{array}\right)^{t} X\left(\begin{array}{l}
A \\
\mathcal{A}
\end{array}\right)+\left(\begin{array}{l}
A \\
\mathcal{A}
\end{array}\right)^{t}\left(\begin{array}{l}
J \\
\mathcal{J}
\end{array}\right),
\end{aligned}
$$

where $A_{a}$ and $J_{a}$ represent the bosonic gauge fields and the bosonic currents, respectively, while $\mathcal{A}_{\alpha}$ and $\mathcal{J}_{\alpha}$ represent the fermionic ones. The matrix $X$ is given by

$$
\begin{aligned}
X & =\left(\begin{array}{cc}
M & P \\
-P^{t} & N
\end{array}\right) \\
& \equiv\left(\begin{array}{cc}
-\epsilon_{a b c} \partial_{c} & \frac{i}{2}\left(C \sigma_{a}\right)_{\alpha \beta} \partial_{\alpha} \\
\frac{i}{2}\left(C \sigma_{b}\right)_{\alpha \beta} \partial_{\beta} & -\frac{i}{2}\left(C \sigma_{a}\right)_{\alpha \beta} \partial_{a}
\end{array}\right) .
\end{aligned}
$$

Because of the existence of the $U(1)$ gauge freedom (4.12), with any smooth function $\Lambda$, the zero-mode equation of $X$ holds

$$
X \cdot\left(\begin{array}{c}
\partial_{a} \Lambda \\
\partial_{\alpha} \Lambda
\end{array}\right)=0
$$

Then, when we take the inverse of $X$, the $U(1)$ gauge freedom needs to be fixed by restricting the function space in which $X$ does not have its zero-mode. For instance, we may apply the super Lorentz gauge

$$
\partial_{a} A_{a}+C_{\alpha \beta} \partial_{\alpha} A_{\beta}=0,
$$

or the ordinary Lorentz gauge

$$
\partial_{a} A_{a}=0 .
$$

Integrating out the super gauge fields $(A, \mathcal{A})$ in Eq.(4.23), a SUSY extension of the Hopf term is obtained as

$$
\begin{aligned}
\mathcal{L}_{\text {sHopf }} & =-\frac{1}{2 \kappa}\left(\begin{array}{l}
J \\
\mathcal{J}
\end{array}\right)^{t} \frac{1}{X}\left(\begin{array}{c}
J \\
-\mathcal{J}
\end{array}\right) \\
& =-\frac{1}{2 \kappa}\left(\begin{array}{l}
J \\
\mathcal{J}
\end{array}\right)^{t}\left(\begin{array}{cc}
\tilde{M} & \tilde{M} P \frac{1}{N} \\
\frac{1}{N} P^{t} \tilde{M} & -\tilde{N}
\end{array}\right)\left(\begin{array}{l}
J \\
\mathcal{J}
\end{array}\right) \\
& =-\frac{1}{2 \kappa}\left(\begin{array}{l}
J \\
\mathcal{J}
\end{array}\right)^{t}\left(\begin{array}{cc}
\tilde{M} & \frac{1}{M} P \tilde{N} \\
\tilde{N} P^{t} \frac{1}{M} & -\tilde{N}
\end{array}\right)\left(\begin{array}{l}
J \\
\mathcal{J}
\end{array}\right),
\end{aligned}
$$


where $\tilde{M}, \tilde{N}$ are given by

$$
\begin{aligned}
\tilde{M} & =\frac{1}{M+P \frac{1}{N} P^{t}}, \\
\tilde{N} & =\frac{1}{N+P^{t} \frac{1}{M} P} .
\end{aligned}
$$

See also the formulas (A6) and (A8) about the inverse of the supermatrix. Expanding $\tilde{M}$ and $\tilde{N}$ in terms of Grassmann odd quantity $P, \mathcal{L}_{\text {sHopf }}$ is simply rewritten as

$$
\begin{aligned}
& \mathcal{L}_{\text {sHopf }} \\
& =-\frac{1}{2 \kappa}\left(\begin{array}{c}
J \\
\mathcal{J}
\end{array}\right)^{t}\left(\begin{array}{cc}
\frac{1}{M}-\frac{1}{M} P \frac{1}{N} P^{t} \frac{1}{M} & \frac{1}{M} P \frac{1}{N} \\
\frac{1}{N} P^{t} \frac{1}{M} & -\frac{1}{N}+\frac{1}{N} P^{t} \frac{1}{M} P \frac{1}{N}
\end{array}\right)\left(\begin{array}{l}
J \\
\mathcal{J}
\end{array}\right) .
\end{aligned}
$$

Because of the nilpotency of the Grassmann number, terms more than quadratic about $P$ do not appear in the expansion. In the original derivative expression, block components of $X^{-1}$ are represented as

$$
\begin{aligned}
& \left(\frac{1}{M}-\frac{1}{M} P \frac{1}{N} P^{t} \frac{1}{M}\right)_{a b} \\
& =\left(\frac{\epsilon \partial}{\partial^{2}}\right)_{a b}-\frac{1}{2}\left(\frac{\epsilon \partial}{\partial^{2}}\right)_{a b}^{3} \frac{\partial}{\partial \theta} C \frac{\partial}{\partial \theta}, \\
& \left(-\frac{1}{N}+\frac{1}{N} P^{t} \frac{1}{M} P \frac{1}{N}\right)_{\alpha \beta} \\
& =2 i\left(\sigma_{a} C\right)_{\alpha \beta} \frac{\partial_{a}}{\partial^{2}}-i\left(\left(\frac{\sigma_{a} \partial_{a}}{\partial^{2}}\right)^{3} C\right)_{\alpha \beta} \frac{\partial}{\partial \theta} C \frac{\partial}{\partial \theta}, \\
& \left(\frac{1}{M} P \frac{1}{N}\right)_{a \alpha}=-\epsilon_{a b c}\left(\sigma_{d} \sigma_{b}\right)_{\alpha \beta} \frac{\partial_{c}}{\partial^{2}} \frac{\partial_{d}}{\partial^{2}} \partial_{\beta}, \\
& \left(\frac{1}{N} P^{t} \frac{1}{M}\right)_{\alpha a}=-\epsilon_{a b c}\left(\sigma_{d} \sigma_{b}\right)_{\alpha \beta} \frac{\partial_{d}}{\partial^{2}} \frac{\partial_{c}}{\partial^{2}} \partial_{\beta},
\end{aligned}
$$

where $\frac{1}{\partial_{a}}=\frac{\partial_{a}}{\partial^{2}}, \partial^{2}=\partial_{a}^{2}, \delta^{2}(\theta)=\frac{1}{2} \theta C \theta,(\epsilon \partial)_{a b}=\epsilon_{a b c} \partial_{c}$ and we used the Lorentz gauge (4.27) to obtain $\frac{1}{\epsilon \partial}=$ $-\frac{\epsilon \partial}{\partial^{2}}$. The identity matrix 1 corresponds to $\delta^{3}(x) \delta^{2}(\theta)$ in the function sense. Since each inverse matrix performs one integration in the parameter space, the SUSY Hopf term is expressed by highly non-local interactions. For instance, the second term on the right-hand side in Eq.(4.31a) contains $\left(\epsilon \partial / \partial^{2}\right)^{3}$, which performs $x$-space integration 3 times. The fermionic terms in $\mathcal{L}_{s H o p f}$ are regarded as newly induced phases by the fermionic gauge field $\mathcal{A}$. As the topological charge of the supermonopole is given by that of its bosonic counterpart [20], we may define the knot invariant of the SUSY Hopf term by its original Hopf term which appears as the first term in Eq.(4.31a).

\section{SUSY Maxwell-Chern-Simons theory and topological masses}

The inner product of the super vector field strengths $\left(F_{a}, F_{\alpha}\right)$ gives an $O S p(1 \mid 2)$ singlet

$$
\begin{aligned}
\mathcal{L}_{s M}= & -\frac{1}{2 e^{2}}\left(F_{a}^{2}+C_{\alpha \beta} F_{\alpha} F_{\beta}\right) \\
= & -\frac{1}{4 e^{2}}\left(F_{a b}^{2}+\frac{i}{2} \epsilon_{a b c}\left(C \sigma_{c}\right)\left(F_{a b} F_{\alpha \beta}+F_{a \alpha} F_{b \beta}\right)\right. \\
& \left.+\frac{1}{2} C_{\alpha \beta} F_{a \alpha} F_{a \beta}+\frac{1}{4} C_{\alpha \beta} C_{\gamma \delta} F_{\alpha \gamma} F_{\beta \delta}\right),
\end{aligned}
$$

which we adopt as the SUSY Maxwell Lagrangian. The SUSY Maxwell-CS Lagrangian is constructed by the coupling of $\mathcal{L}_{s M}$ and $\mathcal{L}_{s C S}$ :

$$
\mathcal{L}_{s M C S}=-\frac{1}{2 e^{2}}\left(F_{a}^{2}+C_{\alpha \beta} F_{\alpha} F_{\beta}\right)+\frac{\kappa}{2}\left(A_{a} F_{a}+A_{\alpha} F_{\alpha}\right) .
$$

The equations of motion are derived as

$$
\begin{aligned}
& \frac{1}{e^{2}} \partial_{b} F_{b a}-i \frac{3}{8 e^{2}} \epsilon_{a b c}\left(C \sigma_{c}\right)_{\alpha \beta} \partial_{b} F_{\alpha \beta}-\frac{1}{4 e^{2}} C_{\alpha \beta} \partial_{\alpha} F_{a \beta} \\
& \quad=\kappa \epsilon_{a b c} F_{b c}+i \frac{\kappa}{2}\left(C \sigma_{a}\right)_{\alpha \beta} F_{\alpha \beta}, \\
& i \frac{3}{8 e^{2}} \epsilon_{a b c}\left(C \sigma_{c}\right)_{\alpha \beta} \partial_{\beta} F_{a b}-\frac{1}{4 e^{2}} C_{\alpha \beta}\left(\partial_{a} F_{a \beta}+C_{\gamma \delta} \partial_{\gamma} F_{\beta \delta}\right) \\
& \quad=i \kappa\left(C \sigma_{a}\right)_{\alpha \beta} F_{a \beta} .
\end{aligned}
$$

With the use of $\left(F_{a}, F_{\alpha}\right)$, Eqs.(4.34) are simply rewritten as

$$
\begin{aligned}
& \frac{1}{e^{2}} \epsilon_{a b c} \partial_{b} F_{c}-\frac{i}{2 e^{2}}\left(C \sigma_{a} C\right)_{\alpha \beta} \partial_{\alpha} F_{\beta}=-2 \kappa F_{a}, \\
& \frac{i}{2 e^{2}}\left(C \sigma_{a} C\right)_{\alpha \beta} \partial_{a} F_{\beta}+\frac{i}{2 e^{2}}\left(C \sigma_{a}\right)_{\alpha \beta} \partial_{\beta} F_{a}=-2 \kappa F_{\alpha} .
\end{aligned}
$$

It is well known that the CS term coupled to the Maxwell Lagrangian induces a topological mass to gauge fields. The mechanism is intuitively explained with the use of the analogy between the Maxwell-CS mechanics and the particle mechanics under magnetic field [25]. The CS term acts as "Lorentz force" in Maxwell-CS mechanics, and yields a cyclotron frequency which corresponds to the gauge mass in Maxwell-CS field theory. Here, we perform similar analyses in the SUSY Maxwell-CS theory. For this end, we treat the 3rd bosonic coordinate as the temporal direction, and work in the super space-time. In a low energy limit, the spatial derivatives of the gauge fields are dropped and the super vector field strengths are reduced to

$$
F_{i} \rightarrow-\epsilon_{i j} \dot{A}_{j}, \quad F_{\alpha} \rightarrow \frac{i}{2}\left(\sigma_{1}\right)_{\alpha \beta} \dot{A}_{\beta},
$$

and the Lagrangian for the SUSY Maxwell-CS mechanics is obtained as

$$
\begin{aligned}
L_{s M C S}= & \frac{1}{2 e^{2}} \dot{A}_{i}^{2}+\frac{1}{8 e^{2}} C_{\alpha \beta} \dot{A}_{\alpha} \dot{A}_{\beta} \\
& -\frac{\kappa}{2} \epsilon_{i j} A_{i} \dot{A}_{j}+\frac{\kappa}{4} i\left(\sigma_{1}\right)_{\alpha \beta} A_{\alpha} \dot{A}_{\beta} .
\end{aligned}
$$


The equations of motion (4.34) become

$$
\begin{aligned}
& \ddot{A}_{i}=e^{2} \kappa \epsilon_{i j} \dot{A}_{j}, \\
& \ddot{A}_{\alpha}=-2 i e^{2} \kappa\left(\sigma_{3}\right)_{\alpha \beta} \dot{A}_{\beta} .
\end{aligned}
$$

$L_{s M C S}$ is formally equivalent to the one-particle Lagrangian on the superplane (3.12) with replacement

$$
\begin{aligned}
& \left(A_{i}, A_{\alpha}\right) \rightarrow\left(x_{i}, 2 C_{\alpha \beta} \theta_{\beta}\right), \\
& e^{2} \rightarrow 1 / m, \\
& \kappa \rightarrow-B .
\end{aligned}
$$

From Eqs.(4.38), the cyclotron frequencies in SUSY Maxwell-CS mechanics are given by $\omega_{B}=e^{2} \kappa$ and $\omega_{F}=2 e^{2} \kappa$, which, respectively, correspond to the topological masses for the bosonic and the fermionic gauge fields in the SUSY Maxwell-CS field theory.

\section{THE SUSY CSLG THEORY AND ITS DUAL REPRESENTATION}

In this section, we present an effective field theory for SUSY QHE. We explore the charge-flux duality in the SUSY QH system, and construct its dual description, where topological solitons arise as fundamental excitations. Based on the dual SUSY CSLG description, we discuss physical properties of topological solitons on the SUSY QH liquid. Since we deal with the spinless matter fields, the topological solitons are realized as the SUSY extension of vortices.

\section{A. Charge-flux duality}

For the existence of the charge-flux duality, space(time) dimension is crucial. In general dimensions, the field strengths behave as 2-rank antisymmetric tensor, while in 3-dimensional space, thanks to the existence of the 3-rank antisymmetric tensor, the 2-rank field strengths are mapped to the 3 -vector field strengths

$$
F_{a}=\frac{1}{2} \epsilon_{a b c} F_{b c} .
$$

Then, in 3-dimensional space, there exists one-to-one correspondence, called the charge-flux duality, between 3vector currents and 3 -vector field strengths

$$
J_{a} \leftrightarrow F_{a}=\frac{1}{2} \epsilon_{a b c} F_{b c},
$$

or, in their components,

$$
\left(\rho, J_{i}\right) \leftrightarrow\left(B, E_{i}\right) .
$$

In the SUSY case, there exists an analogous correspondence between super vector currents and super vector field strengths:

$$
\begin{aligned}
J_{a} & \leftrightarrow F_{a}=\frac{1}{2} \epsilon_{a b c} F_{b c}+i \frac{1}{4}\left(C \sigma_{a}\right)_{\alpha \beta} F_{\alpha \beta}, \\
J_{\alpha} \leftrightarrow F_{a} & =-\frac{i}{2}\left(C \sigma_{a}\right)_{\alpha \beta} F_{a \beta} .
\end{aligned}
$$

Based on this one-to-one relation, we introduce the dual CS field strengths $\left(\tilde{F}_{a}, \tilde{F}_{\alpha}\right)$

$$
\begin{aligned}
\tilde{F}_{a} & =\frac{1}{2} \epsilon_{a b c} \tilde{F}_{b c}+i \frac{1}{4}\left(C \sigma_{a}\right)_{\alpha \beta} \tilde{F}_{\alpha \beta}, \\
\tilde{F}_{\alpha} & =-\frac{i}{2}\left(C \sigma_{a}\right)_{\alpha \beta} \tilde{F}_{a \beta},
\end{aligned}
$$

to match the super matter currents

$$
\begin{gathered}
\tilde{F}_{a}=-J_{a}, \\
\tilde{F}_{\alpha}=-J_{\alpha} .
\end{gathered}
$$

Since $\left(\tilde{F}_{a}, \tilde{F}_{\alpha}\right)$ satisfy the super Bianchi identity $\partial_{a} \tilde{F}_{a}+$ $\partial_{\alpha} \tilde{F}_{\alpha}=0$, Eqs.(5.6) are compatible with the current conservation $\partial_{a} J_{a}+\partial_{\alpha} J_{\alpha}=0$. With the use of the original and the dual CS fields, the SUSY CS Lagrangian is expressed as

$$
\begin{aligned}
\mathcal{L} & =\mathcal{L}_{s C S}+\mathcal{L}_{I} \\
& =\frac{\kappa}{2}\left(A_{a} F_{a}+A_{\alpha} F_{\alpha}\right)-A_{a} \tilde{F}_{a}-A_{\alpha} \tilde{F}_{\alpha} \\
& =\frac{\kappa}{4}\left(\epsilon_{a b c} A_{a} F_{b c}-i\left(C \sigma_{a}\right)_{\alpha \beta} A_{\alpha} F_{a \beta}+\frac{i}{2}\left(C \sigma_{a}\right)_{\alpha \beta} A_{a} F_{\alpha \beta}\right) \\
& -\frac{1}{2} \epsilon_{a b c} A_{a} \tilde{F}_{b c}+\frac{i}{2}\left(C \sigma_{a}\right)_{\alpha \beta} A_{\alpha} \tilde{F}_{a \beta}-\frac{i}{4}\left(C \sigma_{a}\right)_{\alpha \beta} A_{a} \tilde{F}_{\alpha \beta} .
\end{aligned}
$$

Integrating out the original CS fields $\left(A_{a}, A_{\alpha}\right)$, we obtain the dual CS Lagrangian

$$
\begin{aligned}
& \tilde{\mathcal{L}}_{s C S}=-\frac{1}{2 \kappa}\left(\tilde{A}_{a} \tilde{F}_{a}+\tilde{A}_{\alpha} \tilde{F}_{\alpha}\right) \\
& \quad=-\frac{1}{4 \kappa}\left(\epsilon_{a b c} \tilde{A}_{a} \tilde{F}_{b c}-i\left(C \sigma_{a}\right)_{\alpha \beta} \tilde{A}_{\alpha} \tilde{F}_{a \beta}+\frac{i}{2}\left(C \sigma_{a}\right)_{\alpha \beta} \tilde{A}_{a} \tilde{F}_{\alpha \beta}\right) .
\end{aligned}
$$

Since the CS coupling constant in $\tilde{\mathcal{L}}_{s C S}$ is opposite to that in $\mathcal{L}_{s C S}$, the charge-flux duality is restated as the $s$-dual transformation of CS coupling.

\section{B. Relativistic SUSY CSLG theory}

Before detail discussions of the nonrelativistic CSLG theory for SUSY QH liquid, it would be worthwhile to explore the relativistic formulation of SUSY CSLG theory. We regard the 3rd bosonic axis as the temporal direction, and deal with a covariant SUSY CSLG theory in the Wick-rotated super space-time $\mathbb{R}^{3 \mid 2}$. The essential features of duality can be found in this relativistic formulation. We introduce an complex scalar field coupled 
to the SUSY CS fields as

$$
\begin{aligned}
& \mathcal{L}_{C S L G}=\left(\partial_{a}+i c_{a}\right) \phi^{*} \cdot\left(\partial_{a}-i c_{a}\right) \phi \\
& \quad+C_{\alpha \beta}\left(\partial_{\alpha}+i c_{\alpha}\right) \phi^{*} \cdot\left(\partial_{\beta}-i c_{\beta}\right) \phi \\
& \quad+\frac{\kappa}{8 \pi}\left(\epsilon_{a b c} c_{a} f_{b c}-i\left(C \sigma_{a}\right)_{\alpha \beta} c_{\alpha} f_{a \beta}+\frac{i}{2}\left(C \sigma_{a}\right)_{\alpha \beta} c_{a} f_{\alpha \beta}\right),
\end{aligned}
$$

where we denote the SUSY CS fields as $\left(c_{a}, c_{\alpha}\right)$, and their field strengths as $\left(f_{a b}, f_{a \alpha}, f_{\alpha \beta}\right)$. The field equation for $\phi$ is given by

$$
\left(\partial_{a}-i c_{a}\right)^{2} \phi+C_{\alpha \beta}\left(\partial_{\alpha}-i c_{\alpha}\right)\left(\partial_{\beta}-i c_{\beta}\right) \phi=0 .
$$

We decompose the complex scalar field into the density part $\rho$ and the phase part $\chi$ as

$$
\phi=\sqrt{\rho} \chi .
$$

With the use of this decomposition, the kinetic term for $\phi$ is rewritten as

$$
\begin{aligned}
\mathcal{L}_{K} & =\rho\left[\left(i \chi^{*}\left(\partial_{a}-i c_{a}\right) \chi\right)^{2}\right. \\
& \left.+C_{\alpha \beta}\left(i \chi^{*}\left(\partial_{\alpha}+i c_{\alpha}\right) \chi \cdot i \chi^{*}\left(\partial_{\beta}-i c_{\beta}\right) \chi\right)\right],
\end{aligned}
$$

where we postulated the density fluctuations are very small. With the use of the Stratonovich-Hubbard transformation formula [27], $\mathcal{L}_{K}$ is expressed as

$$
\begin{aligned}
\mathcal{L}_{K} & =\left(i \chi^{*}\left(\partial_{a}-i c_{a}\right) \chi\right) \cdot J_{a}+\left(i \chi^{*}\left(\partial_{\alpha}-i c_{\alpha}\right) \chi\right) \cdot J_{\alpha} \\
& -\frac{1}{4 \rho}\left(J_{a}^{2}+C_{\alpha \beta} J_{\alpha} J_{\beta}\right)
\end{aligned}
$$

where $\left(J_{a}, J_{\alpha}\right)$ are auxiliary fields. If there were not CS fields, the equations of motion for $\left(J_{a}, J_{\alpha}\right)$ would be given by

$$
\begin{aligned}
& J_{a}=i \phi^{*} \overleftrightarrow{\partial_{a}} \phi \\
& J_{\alpha}=i C_{\alpha \beta} \phi^{*} \overleftrightarrow{\partial_{\beta}} \phi
\end{aligned}
$$

Thus, $\left(J_{a}, J_{\alpha}\right)$ are essentially the $U(1)$ conserved currents. From Eq.(5.9), the equations of motion for $\left(c_{a}, c_{\alpha}\right)$ are given by

$$
\begin{aligned}
& J_{a}=\frac{\kappa}{2 \pi} f_{a}, \\
& J_{\alpha}=\frac{\kappa}{2 \pi} f_{\alpha} .
\end{aligned}
$$

We further decompose the phase part into the smooth part and the singular part (vortex part) $\varphi$ as

$$
\chi=e^{-i \theta} \varphi
$$

and obtain

$$
\begin{aligned}
\mathcal{L}_{K} & =\left(\partial_{a} \theta+i \varphi^{*} \partial_{a} \varphi+c_{a}\right) J_{a}+\left(\partial_{\alpha} \theta+i \varphi^{*} \partial_{\alpha} \varphi+c_{\alpha}\right) J_{\alpha} \\
& -\frac{1}{4 \rho}\left(J_{a}^{2}+C_{\alpha \beta} J_{\alpha} J_{\beta}\right) .
\end{aligned}
$$

Integrating out the smooth function $\theta$, we find the current conservation law $\partial_{a} J_{a}+\partial_{\alpha} J_{\alpha}=0$. We introduce the dual CS fields $\left(\tilde{c}_{a}, \tilde{c}_{\alpha}\right)$ based on the relation (5.6), and rewrite the total Lagrangian $\mathcal{L}_{C S L G}$ as

$$
\begin{aligned}
\mathcal{L}_{C S L G} & =2 \pi \tilde{c}_{a} \tilde{J}_{a}+2 \pi \tilde{c}_{\alpha} \tilde{J}_{\alpha}-c_{a} \tilde{f}_{a}-c_{\alpha} \tilde{f}_{\alpha} \\
& +\frac{\kappa}{8 \pi}\left(c_{a} f_{a}+c_{\alpha} f_{\alpha}\right)-\frac{1}{16 \rho}\left(\tilde{f}_{a}^{2}+C_{\alpha \beta} \tilde{f}_{\alpha} \tilde{f}_{\beta}\right),
\end{aligned}
$$

where we used a partial integration, and $\left(\tilde{J}_{a}, \tilde{J}_{\alpha}\right)$ denote the topological currents for vortex

$$
\begin{aligned}
& \tilde{J}_{a}=-\frac{i}{2 \pi} \epsilon_{a b c} \partial_{b}\left(\varphi^{*} \partial_{c} \varphi\right)+\frac{1}{4 \pi}\left(C \sigma_{a}\right)_{\alpha \beta} \partial_{\alpha}\left(\varphi^{*} \partial_{\beta} \varphi\right) \\
& \tilde{J}_{\alpha}=-\frac{1}{4 \pi}\left(C \sigma_{a}\right)_{\alpha \beta}\left[\partial_{a}\left(\varphi^{*} \partial_{\beta} \varphi\right)-\partial_{\beta}\left(\varphi^{*} \partial_{a} \varphi\right)\right] .
\end{aligned}
$$

Since $\left(\tilde{J}_{a}, \tilde{J}_{\alpha}\right)$ are topological currents, they automatically satisfy the current conservation law, $\partial_{a} \tilde{J}_{a}+\partial_{\alpha} \tilde{J}_{\alpha}=$ 0 .

In the original bosonic CSLG theory, the explicit representation of the vortex part $\varphi$, is given by $\varphi(x)=\exp (i \alpha(x))$, where $\alpha(x)=\sum_{p} \alpha\left(x-x^{p}\right)=$ $\sum_{p} \arctan \frac{x_{2}-x_{2}^{p}}{x_{1}-x_{1}^{p}}$, and yields the topological charge density

$$
\begin{aligned}
\tilde{\rho}(x) & =-\frac{i}{2 \pi} \epsilon_{i j} \partial_{i}\left(\varphi^{*} \partial_{j} \varphi\right)=-\frac{1}{2 \pi} \epsilon_{i j} \partial_{i} \partial_{j} \alpha(x) \\
& =-\sum_{p} \delta^{2}\left(x-x^{p}\right),
\end{aligned}
$$

where $x^{p}$ denotes the position of the $p$-th vortex. See Ref.[26] for detail discussions. Similarly, in the SUSY case, the vortex part $\varphi$ may be expressed as $\varphi(x, \theta)=$ $\exp (i \alpha(x, \theta))$ with $\alpha(x, \theta)=\sum_{p} \alpha\left(x-x^{p}\right) \delta^{2}\left(\theta-\theta^{p}\right)$, and yields the super topological charge density

$$
\begin{aligned}
\tilde{\rho}(x) & =-\frac{i}{2 \pi} \epsilon_{i j} \partial_{i}\left(\varphi^{*} \partial_{j} \varphi\right)+\frac{1}{4 \pi}\left(\sigma_{1}\right)_{\alpha \beta} \partial_{\alpha}\left(\varphi^{*} \partial_{\beta} \varphi\right) \\
& =-\frac{1}{2 \pi} \epsilon_{i j} \partial_{i} \partial_{j} \alpha(x, \theta)+\frac{1}{2 \pi}\left(\sigma_{1}\right)_{\alpha \beta} \partial_{\alpha} \partial_{\beta} \alpha(x, \theta) \\
& =-\frac{1}{2 \pi} \epsilon_{i j} \partial_{i} \partial_{j} \alpha(x, \theta) \\
& =-\sum_{p} \delta^{2}\left(x-x^{p}\right) \delta^{2}\left(\theta-\theta^{p}\right)
\end{aligned}
$$

Integrating out the original SUSY CS fields $\left(c_{a}, c_{\alpha}\right)$ in Eq.(5.18), the dual SUSY CSLG Lagrangian is derived 
as

$$
\begin{aligned}
\tilde{\mathcal{L}}_{C S L G} \\
=2 \pi \tilde{c}_{a} \tilde{J}_{a}+2 \pi \tilde{c}_{\alpha} \tilde{J}_{\alpha}-\frac{\pi}{2 \kappa}\left(\tilde{c}_{a} \tilde{f}_{a}+\tilde{c}_{\alpha} \tilde{f}_{\alpha}\right) \\
-\frac{1}{16 \rho}\left(\tilde{f}_{a}^{2}+C_{\alpha \beta} \tilde{f}_{\alpha} \tilde{f}_{\beta}\right) \\
=2 \pi \tilde{c}_{a} \tilde{J}_{a}+2 \pi \tilde{c}_{\alpha} \tilde{J}_{\alpha} \\
-\frac{\pi}{2 \kappa}\left(\epsilon_{a b c} \tilde{c}_{a} \tilde{f}_{b c}+i \frac{1}{2}\left(C \sigma_{a}\right)_{\alpha \beta} \tilde{c}_{a} \tilde{f}_{\alpha \beta}-i\left(C \sigma_{a}\right)_{\alpha \beta} \tilde{c}_{\alpha} \tilde{f}_{a \beta}\right) \\
-\frac{1}{8 \rho}\left(\tilde{f}_{a b}^{2}+\frac{i}{2} \epsilon_{a b c}\left(C \sigma_{c}\right)_{\alpha \beta}\left(\tilde{f}_{a b} \tilde{f}_{\alpha \beta}+\tilde{f}_{a \alpha} \tilde{f}_{b \beta}\right)\right. \\
\left.\quad+\frac{1}{2} C_{\alpha \beta} \tilde{f}_{a \alpha} \tilde{f}_{a \beta}+\frac{1}{4} C_{\alpha \beta} C_{\gamma \delta} \tilde{f}_{\alpha \gamma} \tilde{f}_{\beta \delta}\right) .
\end{aligned}
$$

Thus, $\tilde{\mathcal{L}}_{C S L G}$ is equivalent to the SUSY Maxwell-CS Lagrangian coupled to topological currents. The equations of motion for $\left(\tilde{c}_{a}, \tilde{c}_{\alpha}\right)$ are derived as

$$
\begin{aligned}
& \frac{1}{2} \epsilon_{a b c} \partial_{b} \tilde{f}_{c}+\frac{i}{4}\left(C \sigma_{a}\right)_{\alpha \beta} \partial_{\alpha} \tilde{f}_{\beta}-\frac{2 \rho \pi}{\kappa} \tilde{f}_{a}=-4 \rho \pi \tilde{J}_{a} \\
& -\frac{i}{4}\left(\sigma_{a}\right)_{\alpha \beta} \partial_{a} \tilde{f}_{\beta}+\frac{i}{4}\left(\sigma_{a}\right)_{\alpha \beta} \partial_{\beta} \tilde{f}_{a}-\frac{2 \rho \pi}{\kappa} \tilde{f}_{\alpha}=-4 \rho \pi \tilde{J}_{\alpha} .
\end{aligned}
$$

In a low energy limit, the higher derivatives in SUSY Maxwell term are neglected, and the dual SUSY CSLG Lagrangian is approximated by the super vortex Lagrangian coupled to the dual SUSY CS fields. Then, replacing vortex with matter, and the CS coupling constant with its inverse, the dual SUSY CSLG Lagrangian has the same form as the original SUSY CSLG Lagrangian. Similarly, in a low energy limit, Eqs.(5.23) are reduced to

$$
\begin{gathered}
\tilde{f}_{a}=2 \kappa \tilde{J}_{a}, \\
\tilde{f}_{\alpha}=2 \kappa \tilde{J}_{\alpha} .
\end{gathered}
$$

Comparing Eqs.(5.24) with Eqs.(5.15), one may find that the relation between charge and flux in the dual SUSY CSLG theory is opposite to that in the original SUSY CSLG theory. It confirms the previous observation that the $s$-dual transformation of the CS coupling corresponds to the charge-flux duality. Such dual feature has already been reported in the study of the original bosonic CSLG theory [16, 17], and our SUSY CSLG theory shares it. This may be considered as a manifestation that our SUSY CSLG theory provides a natural SUSY framework for the original CSLG theory.

\section{Nonrelativistic CSLG theory for SUSY QH liquid}

Here, we work in the super space-time again, and construct the nonrelativistic SUSY CSLG theory on the superplane. The magnetic field and the electric fields are defined by the super vector fields $\left(F_{a}, F_{\alpha}\right)$ as $B=$ $-F_{t}, E_{i}=F_{i}(i=1,2), E_{\alpha}=F_{\alpha}$. Since the original QH liquid is described by composite bosons coupled to CS fields, it would be reasonable to adopt the following nonrelativistic CSLG Lagrangian as the effective field theoretical description for the SUSY QH liquid

$$
\begin{aligned}
\mathcal{L}_{C S L G}^{n r} & =\phi^{*}\left(i \partial_{t}+\delta A_{t}\right) \phi \\
& -\frac{1}{2 m}\left(-i \partial_{i}+\delta A_{i}\right) \phi^{*} \cdot\left(i \partial_{i}+\delta A_{i}\right) \phi \\
& -\frac{1}{2 m} C_{\alpha \beta}\left(-i \partial_{\alpha}+\delta A_{\alpha}\right) \phi^{*} \cdot\left(i \partial_{\beta}+\delta A_{\beta}\right) \phi \\
& -\frac{1}{2} \int \delta \rho \cdot V \cdot \delta \rho \\
& +\frac{\nu}{8 \pi}\left(\epsilon_{a b c} c_{a} f_{b c}-i\left(C \sigma_{a}\right)_{\alpha \beta} c_{\alpha} f_{a \beta}+\frac{i}{2}\left(C \sigma_{a}\right)_{\alpha \beta} c_{a} f_{\alpha \beta}\right),
\end{aligned}
$$

where $\phi$ denotes the composite boson field, $\delta \rho=\rho-\bar{\rho}$ is the net charge on the background, $\nu=2 \pi \bar{\rho} / B$ is the filling factor, and $\left(\delta A_{a}, \delta A_{\alpha}\right)$ are the effective gauge fields for composite bosons

$$
\begin{aligned}
& \delta A_{a}=A_{a}-c_{a}, \\
& \delta A_{\alpha}=A_{\alpha}-c_{\alpha},
\end{aligned}
$$

where $\left(A_{a}, A_{\alpha}\right)$ are the external gauge fields.

Since $\mathcal{L}_{C S L G}^{n r}$ does not possess a quadratic term about time derivative, the Stratonovich-Hubbard transformations are applied only to quadratic terms about the (super)spatial derivatives. Except for this step, following procedures similar to Sec.VB, the dual nonrelativistic SUSY CSLG Lagrangian is derived as

$$
\begin{aligned}
& \tilde{\mathcal{L}}_{C S L G}^{n r}=2 \pi\left(-\tilde{c}_{t} \tilde{J}_{t}+\tilde{c}_{i} \tilde{J}_{i}+\tilde{c}_{\alpha} \tilde{J}_{\alpha}\right) \\
& -\frac{\pi}{2 \nu}\left(\epsilon^{a b c} \tilde{c}_{a}\left(\tilde{f}_{b c}+\frac{\nu}{\pi} F_{b c}\right)-i\left(C \sigma^{a}\right)_{\alpha \beta} \tilde{c}_{\alpha}\left(\tilde{f}_{a \beta}+\frac{\nu}{\pi} F_{a \beta}\right)\right. \\
& \left.\quad+\frac{i}{2}\left(C \sigma^{a}\right)_{\alpha \beta} \tilde{c}_{a}\left(\tilde{f}_{\alpha \beta}+\frac{\nu}{\pi} F_{\alpha \beta}\right)\right) \\
& -\frac{m}{2 \rho}\left(\tilde{f}_{t i}^{2}+\frac{i}{2} \epsilon_{i j}\left(C \sigma_{i}\right)_{\alpha \beta} \tilde{f}_{t j} \tilde{f}_{\alpha \beta}\right. \\
& \left.\quad+\frac{1}{16}\left(C \sigma_{i}\right)_{\alpha \beta}\left(C \sigma_{i}\right)_{\gamma \delta} \tilde{f}_{\alpha \beta} \tilde{f}_{\gamma \delta}+\frac{1}{4}\left(C \sigma^{a} \sigma^{b}\right)_{\alpha \beta} \tilde{f}_{a \alpha} \tilde{f}_{b \beta}\right) \\
& -\frac{1}{2} \int \delta \rho \cdot V \cdot \delta \rho,
\end{aligned}
$$

where $\delta \rho$ is given by $\delta \rho=\frac{1}{2} \epsilon_{i j}\left(\tilde{f}_{i j}+\frac{\nu}{2 \pi} F_{i j}\right)+$ $\frac{i}{4}\left(\sigma_{1}\right)_{\alpha \beta}\left(\tilde{f}_{\alpha \beta}+\frac{\nu}{2 \pi} F_{\alpha \beta}\right)$. Further, we integrate out $\tilde{c}_{t}$ to obtain an effective action for super vortices. The equation of motion for $\tilde{c}_{t}$ is given by

$\left(\partial_{i}^{2}+\frac{1}{4} C_{\alpha \beta} \partial_{\alpha} \partial_{\beta}\right) \tilde{c}_{t}=-\frac{2 \pi \rho}{m}\left(\tilde{\rho}-\frac{1}{\nu} \delta \rho\right)+($ higher derivatives $)$,

where $\tilde{\rho}=-\tilde{J}_{t}$. Eliminating $\tilde{c}_{t}$ in Eq.(5.27), the density excess is given by

$$
\delta \rho=\nu \tilde{\rho} .
$$


This relation suggests that the super vortex with unit topological number carries the fractional electric charge $\nu$. Inserting Eq.(5.29) to $\tilde{\mathcal{L}}_{C S L G}^{n r}$ and extracting vortex part, the effective Lagrangian for the super vortex is obtained as

$$
\tilde{\mathcal{L}}_{\text {eff }}=2 \pi\left(\tilde{c}_{i} \tilde{J}_{i}+\tilde{c}_{\alpha} \tilde{J}_{\alpha}\right)+\nu A_{t} \tilde{\rho}-\frac{1}{2} \nu^{2} \int \tilde{\rho} \cdot V \cdot \tilde{\rho} .
$$

As expected, the super vortex is coupled to the electric potential with coupling $\nu$. In a low energy limit in which super vortices are approximated as point-like objects, $\tilde{\mathcal{L}}_{\text {eff }}$ is written as

$$
\tilde{L}_{e f f}=2 \pi \sum_{p}\left(\tilde{c}_{i}^{p} \dot{x}_{i}^{p}+\tilde{c}_{\alpha}^{p} \dot{\theta}_{\alpha}^{p}\right)-V
$$

where $\left(x_{i}^{p}, \theta_{\alpha}^{p}\right)$ denotes the position of the $p$-th super vortex, and $V$ represents electric interactions between super vortices. Since $\tilde{L}_{\text {eff }}$ is formally equivalent to the charged particle Lagrangian (3.1) with $m=0$, the super vortex motion is similar to the particle motion in the LLL. The equations of motion for super vortex are derived as

$$
\begin{aligned}
& 2 \pi\left(-\tilde{f}_{i j} \dot{x}_{j}+\tilde{f}_{i \alpha} \dot{\theta}_{\alpha}\right)=\mathcal{E}_{i}, \\
& 2 \pi\left(\tilde{f}_{i \alpha} \dot{x}_{i}+\tilde{f}_{\alpha \beta} \dot{\theta}_{\beta}\right)=C_{\alpha \beta} \mathcal{E}_{\beta},
\end{aligned}
$$

where $\mathcal{E}_{i}=-\partial_{i} V$ and $\mathcal{E}_{\alpha}=C_{\alpha \beta} \partial_{\beta} V$. From these equations, we obtain the super Hall law for vortex

$$
\mathcal{E}_{i} \dot{x}_{i}+C_{\alpha \beta} \mathcal{E}_{\alpha} \dot{\theta}_{\beta}=0
$$

Thus, the super vortex moves perpendicularly to the direction in which it is pushed.

\section{SUMMARY AND DISCUSSIONS}

We studied SUSY extensions of the CS theory and the effective field theory for the SUSY QHE. First, a Lagrangian formalism for the one-particle on the supersphere in the supermonopole background was explored. The particle motion exhibits a SUSY generalization of that on the bosonic sphere in the Dirac monopole background, for instance, Hall orthogonality, cyclotron motion and noncommutative geometry. Next, we constructed a SUSY CS theory with $O S p(1 \mid 2)$ global supersymmetry. Our SUSY CS theory contains many analogous properties peculiar to the original CS theory, such as $U(1)$ gauge symmetry, topological mass generation and etc. In particular, we derived a SUSY generalization of the Hopf term, which is expressed by highly nonlocal interactions. Finally, the CSLG description for the SUSY QH liquid was presented. Based on the duality between the super matter currents and the super vector field strengths, we derived the dual CSLG theory, in which super vortices are coupled to the dual SUSY Maxwell-CS gauge fields. It was shown that the super vortex carries the fractional charge and the vortex motion is equivalent to that of the charged particle in the super LLL.
The SUSY CS theory discussed in this paper is a global $\operatorname{OSp}(1 \mid 2)$ extension of the simplest CS theory in 3-dimensional space. It would be quite interesting to see SUSY generalizations of the CS theory in higher dimensions. Their constructions may be performed based on the higher dimensional SUSY Lie group, such as $O S p(1 \mid 4)$. Higher dimensional SUSY CS theories may have deep connections with supertwistor theory, supermatrix model in higher dimensions, etc. Because of the incompressible property of $\mathrm{QH}$ liquid, the low energy excitations are confined on the edge, and the $\mathrm{QH}$ dynamics is well described only by the edge states. We hope to report edge excitations in SUSY QH liquids based on the SUSY CSLG theory in a future work. The study of the edge excitations may reveal yet unknown dynamical properties of the SUSY QH liquid. Since, at present, the SUSY QHE provides a unique physical setup whose underlying mathematics are given by the nonanticommutative geometry, it would be quite worthwhile to accomplish full analyses of the SUSY QHE. Such explorations are expected to lead to the deeper understanding of novel physics in the non-anticommutative world.

\section{ACKNOWLEDGEMENTS}

I would like to acknowledge Satoshi Iso for valuable comments. I also thank Masanori Hanada, Masatoshi Sato and Tatsuya Tokunaga for useful conversations. Most of this work was performed during the stay at ISSP and KEK. I appreciate the warm hospitalities in the both institutes.

\section{APPENDIX A: SEVERAL FORMULAS ABOUT SUPERMATRIX}

When the supermatrix $X$ takes the form of

$$
X=\left(\begin{array}{cc}
M & P \\
Q & N
\end{array}\right),
$$

(where $M$ and $N$ are Grassmann even matrices, $P$ and $Q$ are Grassmann odd matrices) its superdeterminant is given by

$$
\operatorname{sdet} X=\frac{\operatorname{det}\left(M-P N^{-1} Q\right)}{\operatorname{det} N}=\frac{\operatorname{det} M}{\operatorname{det}\left(N-Q M^{-1} P\right)},
$$

and the super-adjoint is defined as

$$
X^{\ddagger}=\left(\begin{array}{cc}
M^{\dagger} & -Q^{\dagger} \\
P^{\dagger} & N^{\dagger}
\end{array}\right) .
$$

It is noted that our definition of super-adjoint is different from the conventional one. (In the conventional definition, the minus sign is placed in front of $P^{\dagger}$, not $Q^{\dagger}$.) Similarly, the supertrace is defined as

$$
\operatorname{str}(X)=\operatorname{tr}(M)-\operatorname{tr}(N) .
$$


For instance, for the fundamental representations (2.2), the supertraces are calculated as

$$
\begin{aligned}
& \operatorname{str}\left(l_{a} l_{b}\right)=\frac{1}{2} \delta_{a b}, \\
& \operatorname{str}\left(l_{a} l_{\alpha}\right)=0, \\
& \operatorname{str}\left(l_{\alpha} l_{\beta}\right)=-\frac{1}{2} C_{\alpha \beta} .
\end{aligned}
$$

The inverse of $X$ is given by

$$
\begin{aligned}
X^{-1} & =\left(\begin{array}{cc}
\frac{1}{M-P \frac{1}{N} Q} & -\frac{1}{M-P \frac{1}{N} Q} P \frac{1}{N} \\
-\frac{1}{N} Q \frac{1}{M-P \frac{1}{N} Q} & \frac{1}{N-Q \frac{1}{M} P}
\end{array}\right) \\
& =\left(\begin{array}{cc}
\frac{1}{M-P \frac{1}{N} Q} & -\frac{1}{M} P \frac{1}{N-Q \frac{1}{M} P} \\
-\frac{1}{N-Q \frac{1}{M} P} Q \frac{1}{M} & \frac{1}{N-Q \frac{1}{M} P}
\end{array}\right) .
\end{aligned}
$$

When $X$ takes the special form

$$
X=\left(\begin{array}{cc}
M & P \\
-P^{t} & N
\end{array}\right)
$$

its inverse is calculated as

$$
X^{-1}=\left(\begin{array}{cc}
\tilde{M} & -\tilde{M} P \frac{1}{N} \\
\frac{1}{N} P^{t} \tilde{M} & \tilde{N}
\end{array}\right)=\left(\begin{array}{cc}
\tilde{M} & -\frac{1}{M} P \tilde{N} \\
\tilde{N} P^{t} \frac{1}{M} & \tilde{N}
\end{array}\right),
$$

where $\tilde{M}$ and $\tilde{N}$ are defined in Eqs.(4.29).

\section{APPENDIX B: SUPER JACOBI AND BIANCHI IDENTITIES}

With arbitrary operators $(A, B, C)$ (any of $A, B, C$ can be Grassmann even or odd), it is easy to check the super
Jacobi identities

$$
\begin{aligned}
& {[[A, B], C]+[[B, C], A]+[[C, A], B]=0,} \\
& \{[A, B], C\}+[\{B, C\}, A]-\{[C, A], B\}=0, \\
& {[\{A, B\}, C]+[\{B, C\}, A]+[\{C, A\}, B]=0 .}
\end{aligned}
$$

Substituting $(A, B, C)=\left(D_{a}, D_{b}, D_{c}\right)$ (where $D_{a}=\partial_{a}+$ $i A_{a}$ ) and $(A, B, C)=\left(D_{a}, D_{b}, D_{\alpha}\right)$ (where $D_{\alpha}=\partial_{\alpha}+$ $\left.i A_{\alpha}\right)$ to the 1st Jacobi identity (B1a) respectively, we obtain the 1st Bianchi identity

$$
\partial_{a} F_{b c}+\partial_{b} F_{c a}+\partial_{c} F_{a b}=0
$$

and the 2nd Bianchi identity

$$
\partial_{a} F_{b \alpha}+\partial_{b} F_{\alpha a}+\partial_{\alpha} F_{a b}=0
$$

Similarly, substituting $(A, B, C)=\left(D_{a}, D_{\alpha}, D_{\beta}\right)$ to the 2nd Jacobi identity (B1b), the 3rd Bianchi identity

$$
\partial_{a} F_{\alpha \beta}-\partial_{\alpha} F_{a \beta}-\partial_{\beta} F_{a \alpha}=0
$$

is obtained. From the last identity (B1c), with $(A, B, C)=\left(D_{\alpha}, D_{\beta}, D_{\gamma}\right)$, we obtain the last Bianchi identity

$$
\partial_{\alpha} F_{\beta \gamma}+\partial_{\beta} F_{\gamma \alpha}+\partial_{\gamma} F_{\alpha \beta}=0
$$

[1] Shou-Cheng Zhang, Jiangping Hu, "A Four Dimensional Generalization of the Quantum Hall Effect", Science 294 (2001) 823; cond-mat/0110572.

[2] B.A. Bernevig, J.P. Hu, N. Toumbas, S.C. Zhang, "The Eight Dimensional Quantum Hall Effect and the Octonions", Phys.Rev.Lett. 91 (2003) 236803; condmat/0306045.

[3] Kazuki Hasebe, Yusuke Kimura, "Dimensional Hierarchy in Quantum Hall Effects on Fuzzy Spheres", Phys.Lett.B602(2004) 255-260; hep-th/0310274.

[4] Dimitra Karabali, V.P. Nair, "Quantum Hall Effect in Higher Dimensions", Nucl.Phys. B641 (2002) 533-546; hep-th/0203264.

[5] V.P. Nair, S. Randjbar-Daemi, "Quantum Hall effect on $S^{3}$, edge states and fuzzy $S^{3} / \mathbf{Z}_{2}$ ", Nucl.Phys. B679 (2004) 447-463; hep-th/0309212.

[6] Ahmed Jellal, "Quantum Hall Effect on Higher Dimensional Spaces", Nucl.Phys.B725 (2005) 554-576; hepth/0505095.

[7] Giovanni Landi, "Spin-Hall effect with quantum group symmetry", Lett.Math.Phys.75 (2006) 187-200; hep- th/0504092.

[8] Hirosi Ooguri, Cumrun Vafa, "The CDeformation of Gluino and Non-planar Diagrams", Adv.Theor.Math.Phys. 7 (2003) 53-85, hep-th/0302109; "Gravity Induced C-Deformation", Adv.Theor.Math.Phys. 7 (2004) 405-417, hepth/0303063.

[9] J. de Boer, P. A. Grassi, P. van Nieuwenhuizen, "Noncommutative superspace from string theory", Phys.Lett. B574 (2003) 98-104, hep-th/0302078.

[10] Nathan Seiberg, "Noncommutative Superspace, $N=1 / 2$ Supersymmetry, Field Theory and String Theory", JHEP 0306 (2003) 010, hep-th/0305248.

[11] Kazuki Hasebe, "Supersymmetric Quantum Hall Effect on a Fuzzy Supersphere", Phys.Rev.Lett. 94 (2005) 206802; hep-th/0411137.

[12] Kazuki Hasebe, "Quantum Hall Liquid on a Noncommutative Superplane", Phys.Rev.D72 (2005) 105017; hepth/0503162.

[13] Evgeny Ivanov, Luca Mezincescu, Paul K. Townsend, "Fuzzy $C P(n \mid m)$ as a quantum superspace", hep- 
th/0311159, "A Super-Flag Landau Model", hepth/0404108, "Planar Super-Landau Models", JHEP 0601 (2006) 143; hep-th/0510019.

[14] S. Bellucci, A. Beylin, S. Krivonos, A. Nersessian, E. Orazi, " $N=4$ supersymmetric mechanics with nonlinear chiral supermultiplet", Phys.Lett. B616 (2005) 228-232; hep-th/0503244.

[15] James Gates Jr, Ahmed Jellal, EL Hassan Saidi, Michael Schreiber, "Supersymmetric Embedding of the Quantum Hall Matrix Model", JHEP 0411 (2004) 075; hepth/0410070.

[16] S.C. Zhang, T.H. Hansson, S. Kivelson "An Effective Field Theory Model For The Fractional Quantum Hall Effect", Phys.Rev.Lett.62 (1989) 82-85.

[17] S.C. Zhang, "The Chern-Simons-Landau-Ginzburg Theory Of The Fractional Quantum Hall Effect", Int.J.Mod.Phys. B6 (1992) 25-58.

[18] B.A. Bernevig, C.H. Chern, J.P. Hu, N. Toumbas, S.C. Zhang, "Effective field theory description of the higher dimensional quantum Hall liquid", Annals Phys. 300 (2002) 185; cond-mat/0206164

[19] See, for instance, Gerald V. Dunne, "Aspects of ChernSimons Theory", hep-th/9902115.

[20] Giovanni Landi, "Projective Modules of Finite Type over the Supersphere $S^{2,2}$ ”, Differ.Geom.Appl. 14 (2001) 95-
111; math-ph/9907020.

[21] Kazuki Hasebe, Yusuke Kimura, "Fuzzy Supersphere and Supermonopole", Nucl.Phys. B709 (2005) 94-114; hepth/0409230.

[22] Kiyoshi Ezawa, Atushi Ishikawa, "Osp(1|2) ChernSimons gauge theory as 2D N=1 Induced Supergravity", Phys.Rev. D56 (1997) 2362-2368; hep-th/9612031.

[23] Bum-Hoon Lee, Choonkyu Lee and Hyunsoo Min, "Supersymmetric Chern-Simons vortex systems and fermion zero modes", Phys. Rev. D 45 (1992) 4588-4599.

[24] Satoshi Iso, Hiroshi Umetsu, "Gauge Theory on Noncommutative Supersphere from Supermatrix Model", Phys.Rev. D69 (2004) 105003; hep-th/0311005.

[25] G. V. Dunne, R. Jackiw, and C. A. Trugenberger, "Topological (Chern-Simons) quantum mechanics", Phys. Rev. D 41 (1990) 661-666.

[26] Satoshi Iso, Dimitra Karabali, B. Sakita, "OneDimensional Fermions as Two-Dimensional Droplets Via Chern-Simons Theory ", Nucl.Phys. B388 (1992) 700714; hep-th/9202012.

[27] Introducing the auxiliary fields $\left(J_{a}, J_{\alpha}\right)$, the SUSY quadratic term $V_{a}^{2}+C_{\alpha \beta} V_{\alpha} V_{\beta}$ is rewritten as $V_{a} J_{a}+$ $V_{\alpha} J_{\alpha}-\frac{1}{4}\left(J_{a}^{2}+C_{\alpha \beta} J_{\alpha} J_{\beta}\right)$. 